\title{
Chapter 8 \\ The Surgical Approach to Elderly Patients with HNSCC
}

\author{
Andreas Dietz
}

\section{Introduction}

Recently, some reviews and recommendations regarding treatment of head and neck cancer in elderlies have been published by Petr Szturz, Paolo Bossi and Jan Vermorken [1,2]. These papers point out that the age of 70 (or even 75) as a cut-off defining the elderly has been broadly accepted and adopted by the "National Institute on Aging" and the "National Institutes of Health" [3]. This cut-off point may better capture the reality in terms of biological alterations occurring with advancing age, because aging is associated with a progressive loss of functional reserve of multiple organ systems, increased prevalence of chronic diseases, enhanced susceptibility to stress, and fluctuations in social support and economic resources [4]. Regarding prevalence of head neck surgery, data from New Zealand showed number and age of patients undergoing major head and neck cancer surgery peaked in the age group of 71-80 years [5].

Chronological age does not sufficiently correlate with biological parameters and provides only limited information for personalized management. Therefore Szturz et al. points out that in clinical practice, the crucial step is to distinguish a fit-old individual, who will likely withstand a radical treatment with curative intent, from a frail-old patient, who will probably not tolerate such approach. To deliver optimal patient care at an individual level a team approach represented by a multidisciplinary tumor board is essential. These meetings should offer a collaborative review of each case with special attention to disease factors (site, stage, biology, and risk factors for locoregional or distant relapse), patient factors (age, sex, performance and nutritional status, comorbid conditions, oral health, life-style habits, and socioeconomic background), treatment options, and patient preferences [1, 2]. With

\footnotetext{
A. Dietz $(\bowtie)$

Clinic of Otolaryngology, Head and Neck Surgery and Department of Head Medicine and Oral Health, University of Leipzig, Leipzig, Germany

e-mail: andreas.dietz@medizin.uni-leipzig.de
} 
special focus on indication for primary or secondary (salvage) surgical procedures some specific factors must be taken into consideration.

\section{Functional Physiological Age Related Issues with Impact on Selection for Surgical Treatment}

Physiology of aging is characterized by a couple of differently distinct biologic developments with relevant impact on assessment for feasibility of surgical procedures. To check all these factors would be very time consuming and unrealistic in daily routine. Nevertheless, knowledge about these factors is mandatory and can sharpen the view by checking some representative indices for selecting the right patients (Table 8.1).

There are several factors going along with worse functional outcome after surgery (and other treatments) if not taken into consideration. The upper esophageal sphincter (UES) contraction reflex and the sensitivity of the complex swallowing process mainly at the level of the larynx entrance is reduced in advanced ages (Involvement of cranial nerves like vagus, trigeminus, glossopharyngeus, accessories, hypoglossus and plexus cervicalis composite all structures flexible and mobile). Additionally, the trigger to swallow is reduced and as well as protective mechanisms like coughing or harrumphing. Therefore, the danger of silent or definitive aspiration increases and can cause fatal complications like pneumonia. Kawamura

Table 8.1 Practical factors relevant for surgical indication going along with physiological aging [6-10]

- The healing of skin wounds is significantly prolonged

- The compliance of the cardiovascular system is reduced with resulting in hypertension

- The upper esophageal sphincter (UES) contraction reflex is reduced

- The elasticity of the chest wall is reduced

- There is loss of supporting tissue of the pulmonary airways

- There is an altered thermoregulation due to both changes in muscle and fat mass and a reduced metabolism

- The sympathetic activity is decreased

- The compensatory reaction of the autonomic nervous system to stress and volume losses is reduced

- The tolerance to a reduced number of oxygen carriers ( $\mathrm{Hb}$ value) is reduced

- There is an earlier indication for transfusion than in younger patients

- There is a decreased respiratory drive on hypercapnia and/or hypoxia

- The is a reduced volume of distribution

- There is a decreased hepatic and renal clearance

- There is a higher sensitivity of the central and peripheral nervous system to anesthetics and muscle relaxants

- On average, the need for anesthetics in patients $>80$ years is about $30 \%$ lower than in those aged 20 
et al. could demonstrate, that the frequency elicitation of UES contraction reflex decreases significantly with age while the magnitude of change in UES pressure remains unchanged, indicating a deleterious effect of aging on the afferent arm of this reflex. This reflex is altered in some dysphagia patients [7]. This age-related difference in swallowing function, sensibility for aspiration and successful rehabilitation can play a major role in finding the right surgical procedure, like trade off in favor for total compared to partial laryngectomy in elderlies for instance. Especially in supraglottic laryngectomies the positive correlation between increasing age and the occurrence of aspiration pneumonia should be considered. Therefore, preoperative pulmonary function tests (FEV1, VC) can be helpful to consider risk of postoperative aspiration pneumonia [9]. Compared to the relevance of swallowing the quality of voice is less important and of secondary interest for the late quality of life outcome especially in elderlies.

Equally relevant as one of the most significant predictors for successful outcome after surgery in elderly patients is the preoperative lung function. The 30-day mortality rate following upper-airway and thoracic invasive surgery on average is $6 \%$ in elderly patients. Up to $50 \%$ of the causes of death in these patients are related to pulmonary complications/comorbidities [6]. Improved preoperative preparation (e.g. breathing gymnastics), the development of modern anesthetics as well as the optimized perioperative monitoring (relaxometry, pulse oximetry) have been able to contribute significantly to the fact that the perioperative risk in the old patient is not significantly increased by age itself [6]. In general, pulmonary function decreases with advanced age and can cause major problems if the surgical procedure is not fitting into the performance precondition. Interestingly, restriction of the thorax expansion capacity by delivery of a pectoralis major myocutaneous (PMC) flap (very common procedure in reconstructive head and neck surgery) and tight wound closure of the overlying skin can cause severe problems after surgery. Pulmonary atelectasis has been reported in patients undergoing these procedures, and many of these patients are heavy smokers and drinkers and have associated cardiopulmonary disorders. Flap harvest and donor site closure may lead to impairment of pulmonary function after delivery of pectoralis major myocutaneous (PMC) flap in surgical reconstruction in patients with cancer of the head and neck. Talmi et al. evaluated prospectively patients undergoing extirpation of head and neck tumors with PMC reconstruction. Patient age, smoking history (pack-years), anesthesia duration, percentage predicted pre- and postoperative FEV1, percentage-predicted pre- and postoperative FVC (forced vital capacity), and preoperative $\mathrm{SaO}_{2}$ (oxygen saturation) were evaluated. A series of 11 patients, 5 of whom smoked, could be evaluated postoperatively. Preoperative FEV1/FVC was more than $70 \%$ and FEV1 more than $75 \%$ predicted in all patients. A decrease in FVC was observed in seven of the 11 patients, which ranged between $2 \%$ and $27 \%$ without any clinically obvious respiratory manifestations. A baseline $\mathrm{SaO}_{2}$ of more than $96 \%$ was noted in all patients. Four of nine postoperative chest X-rays demonstrated atelectasis. The authors conclude, that alternative methods of surgical defect closure should be considered in patients with severe preexisting pulmonary disorders [10]. 


\section{Assessment of Comorbidity for Surgical Treatment}

In general prevalence of comorbidities in head and neck cancer patients is of importance, and that is true not only for elderly patients Since chronic abuse of tobacco and alcohol are still the main risk factors for head and neck cancer both factors also cause many other diseases. Pulmonary and cardio-vascular disorders are the main limiting factors for radical and extensive surgical procedures. In elderlies, the mixture of additional age related diseases and tobacco/alcohol related comorbidities could be a complex challenge for indicating the individual treatment in the single patient. Table 8.2 summarizes the frequent surgery-relevant diseases in older age.

Elderly patients (70+ years) have a high prevalence of comorbidity resulting in a high frequency of polypharmacy defined as a daily use of five drugs or more. Jorgensen et al. [11] compared 30,122 cancer cases with 120,485 controls (42.6\% $>70$ years) and found mean drug use of 5.12 in elderlies with cancer 5.12 and 4.07 in controls in general (not specific for head and neck cancer).

In the experience of most head and neck surgeons recovery after extensive surgical procedures is also linked to the age. Patients recovery can be prolonged in elderlies, even when the operation went smoothly. Grammatica et al. published recently a retrospective multi institutional study in the "Older Old (>75)" and "Oldest Old (>85)" undergoing free flaps for advanced oral cancer (the majority of the reconstructions were performed by radial forearm flap and ALT (anterior lateral thigh flap); about $10 \%$ had fibula/scapula flaps). Pre-operative assessment was performed by the American Society of Anesthesiologists (ASA) and the Adult Comorbidity Evaluation 27 (ACE-27) scores. Complications after surgery were grouped as medical or surgical, and major or minor according to the Clavien-Dindo scale. The majority (67\%) of patient met ASA-3 criteria (severe systemic disease), $63.5 \%$ met the ACE-27 score 2 criteria (moderate comorbidity) and $8.3 \%$ had severe comorbidity (ACE-27 score 3). 38\% had a history of smoking and $47.6 \%$ of alcohol abuse. The mean operation time in minutes was 553.5 (range 230-890 min). Overall, 52 $(61.9 \%)$ patients had at least one complication: ASA score, diabetes mellitus, and duration of general anesthesia (DGA) significantly impacted the complication rate at multivariate analysis. Patients with diabetes suffered from $61.1 \%$ complications in contrast to only $31.8 \%$ in non-diabetic patients. $20.2 \%$ of patients had major

Table 8.2 Frequent surgeryrelevant diseases in older age [6]
- Arteriosclerosis

- Lung emphysema

- Chronic obstructive pulmonary disease

- Malnutrition

- Diabetes type II

- Osteoporosis

- Parkinson's disease

- Alzheimer's disease

- Dementia 
surgical complications (11\% flap necrosis). $10.7 \%$ of patients had major medical complications; Smoking and ASA-4 category showed close-to-significance p-values in multivariate analysis. The authors conclude that lengthy DGA (pivotal factor) and in-hospital stay should be carefully considered especially when dealing with advanced age patients. Therefore, surgical teams should be encouraged to reduce the duration of surgery by operating with ablative and reconstruction teams simultaneously. Pre-operative assessment and aggressive management of glycemia in patients with diabetes is mandatory [12].

Keeping an eye on the factor "duration of general anesthesia" which is prognostic for complications in elderlies, some surgical procedures should be balanced by extent of resection, degree of reconstruction and safety. For example, some borderline stages of larynx carcinomas, which could be successfully treated by partial laryngectomy in younger patients but with high risk of long time aspiration in elderlies, could be considered for total laryngectomy or primary chemoradiation. Laryngectomy can be conducted in a short time with minimal trauma by preserving very carefully the surrounding tissues and keeping the operation field very small. Indication management for elective Neck dissection in N0-situations in elderlies can be cautious. Compared to primary chemoradiation this surgical approach is quick, guarantees complete tumor removal und ends up with less late dysphagia for instance.

\section{Age and Special Surgical Procedures}

Although the finding that age in regard of surgical procedures is not an independent negative prognostic factor in head and neck carcinoma patients, there is a widespread mental conflict that suggests that you are better off at being young. Most older patients and their families are more reluctant to agree to major surgical interventions [13]. Overall, there are no prospective randomized studies explicitly addressing age and standard of care in head and neck cancer surgery.

Before the 1960s, the operative mortality rate for elderly patients undergoing elective surgery was two to six times higher than that in the general population [14]. In the early 1970s, McGuirt et al. published the first data addressing head and neck surgical outcome in elderlies [15]. The incidence of both major and minor surgical complications was comparable between the cohorts above and below 70 years of age. However, medical complications, mostly of cardiovascular and pulmonary origin, were higher by $8 \%$ in the elderly subgroup. Perioperative mortality rates, defined as death within 30 days of operation, were 7.4 and $1.4 \%$ in older and younger 70 years subjects, respectively [15]. According to the literature, age "per se" is not an independent contraindication for a surgical intervention in tumors of the head and neck area [16]. Claymen et al. stated in 1998 based on his data in a small retrospective study that although the older age group $(>80)$ had a higher frequency of morbid preoperative conditions, there were no significant differences in perioperative or postoperative complications between the two groups. Careful preoperative staging and evaluation of associated medical illnesses, as well as skillful 
perioperative and postoperative management, are essential for reducing operative morbidity and mortality [17].

In general, all head and neck procedures should be adjusted to the functional status of the individual patient as already mentioned in this chapter. Therefore, the multidisciplinary team should include specialists like phoniatricians, voice specialists and logopedics to asses preoperative swallowing competence and other functional relevant factors. In this context FEES (functional endoscopic evaluation of function) became one of the main investigations as part of the functional staging of the (not only) elderly patient.

Free flap procedures are feasible independent of age, as already mentioned in this chapter (Grammatica). Also, Tarsitano et al. presented data about safety of microsurgical free-tissue transfer and described the rates of major surgical complication being $9 \%$ in young patients and $11 \%$ in elderly patients $(>75)$. They found no significant difference between the two groups in the rates of major and minor flap complications, morbidity or long-term functional outcome [18].

However, the mortality rate in elderly patients has declined in the past 40 years. Today, the overall surgical mortality rate is about $0.9-2.4 \%$, even for patients with cardiac disease, largely as result of safer anesthesia techniques [19]. Both the studies reported by Taristano et al. and Grammatica et al. showed that the ASA score (not age) in multivariate analysis was the only variable associated with an increased complication rate. The perioperative mortality was addressed in a large retrospective study of 810 patients aged over 65 years, where the rate was calculated at $3.5 \%$ [20]. Smaller series later published by other investigators showed similar findings even in the oldest-old category $[1,2]$.

\section{Conclusion}

Head and Neck cancer surgery is feasible independent of age. The preoperative assessment of comorbidity in elderly patient in order to avoid surgical complications is of major importance. Equally relevant as one of the most significant predictors for successful outcome after surgery is the preoperative lung function and the operation time. Close coordination with anesthesia and rapid postoperative mobilization are essential for this. Decision-making and treatment based on specific assessment in an experienced multidisciplinary team is key.

\section{References}

1. Szturz P, Vermorken JB. Treatment of elderly patients with squamous cell carcinoma of the head and neck. Front Oncol. 2016;6:199.

2. Szturz P, Bossi P, Vermorken JB. Systemic treatment in elderly head and neck cancer patients: recommendations for clinical practice. Curr Opin Otolaryngol Head Neck Surg. 2019;27(2):142-50. 
3. Argiris A, Li Y, Murphy BA, Langer CJ, Forastiere AA. Outcome of elderly patients with recurrent or metastatic head and neck cancer treated with cisplatin-based chemotherapy. J Clin Oncol. 2004;22:262-8.

4. Balducci L. Management of cancer in the elderly. Oncology (Williston Park). 2006;20:135-43.

5. Jayakar R, Choi J, MacKinnon C, Tan S. The cost of major head and neck cancer surgery. N Z Med J. 2017;130(1455):111-9.

6. Teymoortash A, Wulf $\mathrm{H}$, Werner JA. Head and neck cancer surgery in the elderly. Laryngorhinootologie. 2002;81(4):293-8.

7. Kawamura O, Easterling C, Aslam M, Rittmann T, Hofmann C, Shaker R. Laryngo-upper esophageal sphincter contractile reflex in humans deteriorates with age. Gastroenterology. 2004;127(1):57-64.

8. Tsaih SW, Korrick S, Schwartz J, Amarasiriwardena C, Aro A, Sparrow D, Hu H. Lead, diabetes, hypertension, and renal function: the normative aging study. Environ Health Perspect. 2004;112(11):1178-82.

9. Cabanillas R, Rodrigo JP, Llorente JL, Suárez V, Ortega P, Suárez C. Functional outcomes of transoral laser surgery of supraglottic carcinoma compared with a transcervical approach. Head Neck. 2004;26(8):653-9.

10. Talmi YP, Benzaray S, Peleg M, Eyal A, Bedrin L, Shoshani Y, Yahalom R, Horowitz Z, Taicher S, Kronenberg J, Shiner RJ. Pulmonary function after pectoralis major myocutaneous flap harvest. Laryngoscope. 2002;112(3):467-71.

11. Jorgensen L, Hallas J, Herrstedt J: Department of Oncology, Odense University Hospital, Institute of Clinical Research, University of Southern Denmark, Odense, Denmark; Institute of Public Health, Research Unit of Clinical Pharmacology, University of Southern Denmark, Odense, Denmark; Odense University Hospital, Odense, Denmark; ASCO 2010.

12. Grammatica A, Piazza C, Pellini R, Montalto N, Lancini D, Vural A, Barbara F, Ferrari M, Nicolai P. Free flaps for advanced oral cancer in the "older old" and "oldest old": a retrospective multi-institutional study. Front Oncol. 2019;9:604.

13. Genden EM, Rinaldo A, Shaha AR, Clayman GL, Werner JA, Suárez C, Ferlito A. Treatment considerations for head and neck cancer in the elderly. J Laryngol Otol. 2005;119(3):169-74.

14. Cole WH. Prediction of operative reserve in the elderly patient. Ann Surg. 1968;168:310.

15. McGuirt WF, Loevy S, McCabe BF, Krause CJ. The risks of major head and neck surgery in the aged population. Laryngoscope. 1977;87:1378-82.

16. Jang IJH, Skanthakumar T, Tan HK, Tan NC, Soo KC, Iyer NG. Elderly patients with advanced head and neck carcinoma: does aggressive treatment result in better outcomes? Otolaryngol Head Neck Surg. 2019;160(4):642-50.

17. Clayman GL, Eicher SA, Sicard MW, Razmpa E, Goepfert H. Surgical outcomes in head and neck cancer patients 80 years of age and older. Head Neck. 1998;20(3):216-23.

18. Tarsitano A, Pizzigallo A, Sgarzani R, Oranges CM, Cipriani R, Marchetti C. Head and neck cancer in elderly patients: is microsurgical free-tissue transfer a safe procedure? Acta Otorhinolaryngol Ital. 2012;32(6):371-5.

19. Foster ED, Davis KB, Carpenter JA, et al. Risk of noncardiac operation in patients with defined coronary disease. Ann Thorac Surg. 1986;41:42-50.

20. Morgan RF, Hirata RM, Jaques DA, Hoopes JE. Head and neck surgery in the aged. Am J Surg. 1982;144:449-51. 
Open Access This chapter is licensed under the terms of the Creative Commons Attribution 4.0 International License (http://creativecommons.org/licenses/by/4.0/), which permits use, sharing, adaptation, distribution and reproduction in any medium or format, as long as you give appropriate credit to the original author(s) and the source, provide a link to the Creative Commons license and indicate if changes were made.

The images or other third party material in this chapter are included in the chapter's Creative Commons license, unless indicated otherwise in a credit line to the material. If material is not included in the chapter's Creative Commons license and your intended use is not permitted by statutory regulation or exceeds the permitted use, you will need to obtain permission directly from the copyright holder. 